\title{
Las Actitudes Frente a la Matemática y la Historia de la Matemática
}

\author{
Attitudes Against Mathematics And History Of Mathematics
}

\author{
Johnny Osman Pérez Carpena
}

\section{RESUMEN}

Objetivo: Establecer el nivel de desarrollo de actitudes frente a la Matemática por medio de la Historia de la Matemática en estudiantes de la escuela profesional de Medicina de la Universidad Continental - Lima en el semestre académico 2019-II. Material y métodos: Los instrumentos utilizados se basaron en encuestas para el Pretest como para el Postest, en la medición de ambas variables. La validación de instrumentos se realizó a través de la evaluación de juicio de expertos. La confiabilidad se aseguró por medio del coeficiente Alfa de Cronbach. El método de la investigación fue de deductivo, de tipo experimental. El diseño fue cuasiexperimental con evaluación de progreso, donde se aplica dos grupos de estudio: Grupo experimental, y grupo control, el nivel de la investigación es de presencia y ausencia de la variable independiente. Resultado: Se evaluó a los estudiantes del primer ciclo de la Escuela Profesional de Medicina en el semestre académico 2019-Il. Se demostró que sí existe una relación significativa entre el nivel de desarrollo de actitudes frente a la Matemática por medio de la Historia de la Matemática en estudiantes de la Escuela Profesional de Medicina de la Universidad Continental - Lima en el semestre académico 2019-Il. Conclusiones: La Historia de la Matemática influye directamente sobre las actitudes frente a la Matemática de una manera significativa, positiva en los estudiantes de la Escuela Profesional de Medicina de la Universidad Continental - Lima en el semestre académico 2019-Il con un coeficiente de significancia bilateral de 0.000 .

Palabras clave: Actitudes, Historia de la matemática, Desarrollo y sesiones.

\section{ABSTRACT}

Objective: To establish the level of development of attitudes towards mathematics through the history of mathematics in students of the professional school of Medicine of the Continental University - Lima in the academic semester 2019-II. Material and methods: The instruments used were based on surveys for the Pretest and for the Posttest, in the measurement of both variables. The validation of instruments was carried out through the evaluation of expert judgment. Reliability was ensured through Cronbach's alpha coefficient. The research method was deductive, experimental. The design was quasi-experimental with progress evaluation, where two study groups are applied: experimental group, and control group, the level of research is the presence and absence of the independent variable. Result: The students of the first cycle of the Professional School of Medicine in the academic semester 2019-II were evaluated. It was shown that there is a significant relationship between the level of development of attitudes towards mathematics through the history of mathematics in students of the professional school of Medicine of the Continental University - Lima in the academic semester 2019-II. Conclusions: The History of Mathematics directly influences Attitudes towards Mathematics in a meaningful, positive way in the students of the Professional School of Medicine of the Continental University - Lima in the academic semester 2019-II with a bilateral significance coefficient of 0.000 .

Keywords: Attitudes, history of mathematics, development and sessions.

\section{INTRODUCCIÒN}

La Matemática es herramienta que no solo abarca el campo científico, sino que es parte de múltiples actividades profesionales. Es por este contexto, que es de importancia poder sentar bases matemáticas fuertes en el estudiantado diversificando en todos los niveles académicos, sobre todo el campo de educación superior. Se le debe prestar mucha atención al aspecto actitudinal pues influye mucho en el aprendizaje. Ahora, con respecto al rendimiento académico superior, existe un suspenso con respecto a esta materia. Si se habla de las causas, será relevante mencionar que se debe a la actitud. Existe una actitud negativa hacia la Matemática. Quizá se debe esto a las estrategias utilizadas, las cuales, no son motivacionales, es decir, no calan en el campo de afectividad de los estudiantes. Ante esa actitud negativa hacia la matemática, entra la Historia de la Matemática, ya que la capacidad crítica del estudiante no se despierta con solo una exposición cerrada y acabada de una ciencia estática, sino que necesita de valores científicos en el sujeto mismo. En este sentido, se busca establecer el vínculo entre las actitudes de las matemáticas y la Historia de la Matemática, pues repercute de manera social. Es por ello que se necesita despertar el interés y la necesidad para asimilar con una mejor actitud hacia la Matemática. De esa manera se podrá adquirir un mejor rendimiento y una mejor preparación para una integración adecuada en la sociedad. Además, se conseguirá un mejor servicio y eso permitirá un beneficio de desarrollo para nuestro país.

Como antecedentes, primero se abordó el aspecto de las actitudes frente a la matemática; ante ello Gómez (2009), menciona que estas se enfocan en la valoración y la estima a esta disciplina y al provecho de esta materia, a su vez por su aprendizaje. Están vinculados a las actitudes en matemáticas, la parte afectiva, la satisfacción, la curiosidad, la valoración, entre otros. Por su parte, Mato, Soneira y Muñoz (2018), lo señalan como manifestaciones de la conducta que tienen sus orígenes en las creencias, las emociones, los hábitos, las experiencias previas. Por último, Mamani (2012), los toma

\section{Recibido:24/01/2020 - Aprobado:03/02/2020}

Este es un artículo de acceso abierto, distribuido bajo los términos de la Licencia Creative Commons Atribución 4.0 Internacional (http://creativecommons.org/licenses/by/4.0/) (a) (1)

1. Docente de la Universidad Nacional Mayor de San Marcos, Perú 
como formas de contemplar, interpretar y actuar sobre el mundo que le rodea a cualquier individuo y de esa manera pueda impregnarse de dichas actitudes aun sin tener presente a las matemáticas como ciencia. Las actitudes en matemáticas involucran tres componentes: el aspecto afectivo, es decir, los sentimientos; el aspecto cognitivo, es decir, las creencias; y el aspecto comportamental, es decir, el que se acerque o se aleje de las matemáticas. En cuanto a la Historia de la Matemática, González (2004), menciona que brinda conocimientos sobre la manera cómo se dieron los variados conceptos matemáticos, sobre las intuiciones y las ideas de donde surgieron; tiene que ver con el origen de los términos, lenguajes y notaciones de manera singular, los variados problemas que se resolvían, los contextos en los cuales se realizaban, las técnicas para desarrollar cualquier situación matemática, los teoremas, las demostraciones, el lineamiento para poder conjeturar teorías, la evolución de la misma ciencia. Por su parte, Vidal (2003), indica que dentro de un aula de clases está vinculada con el aspecto cultural, el aspecto humanístico, el aspecto interdisciplinar y la posibilidad de una organización en cuanto al currículo. Por último, Temple (2016) señala que: "sabios que han sido excelentes pedagogos [Poincaré, Klein, Toeplitz, Köthe, Bell, Courant, Puig Adam, Lakatos, Kline, Santaló...] han ponderado la importancia que tiene la Historia de la Matemática en la calidad de su enseñanza." (pág. 18). En este sentido, muchos pedagogos han mencionado la importancia que tiene la Historia de la Matemática en la mejora de la transmisión de conocimientos matemáticos. En cuanto a la relación de ambas variables, González(2004), lo sindica como un medio para desarrollar actitudes en la matemática y que constituye una de las principales manifestaciones del pensamiento que han dejado huella a través de la humanidad y que está vinculado a los grandes hitos del conocimiento y además de la cultura. La Historia de la Matemática pone de manifiesto una dimensión cultural distinta y que tiene un impacto interesante en la actitud para dicha ciencia. Por su parte, Temple y Puig (2004) mencionan que permite comprender las interrogantes que surgen ante las diversas concepciones, las percepciones y nociones de donde se manifestaron.

Por último, la investigación tuvo como objetivo fundamental establecer el nivel de desarrollo de las actitudes frente a las matemáticas por medio de la Historia de la Matemática en estudiantes de la Escuela Profesional de Medicina de la Universidad Continental - Lima en el semestre académico 2019-II.

\section{MATERIALES Y MÉTODOS}

La investigación es de tipo deductiva donde el tipo de investigación fue experimental, dentro de las investigaciones experimentales, se subclasifica como una investigación cuasiexperimental debido a que las poblaciones o grupos estaban determinados por la división de las secciones.

El diseño de contrastación utilizado en la presente investigación fue el diseño cuasiexperimental con evaluación de progreso, donde se aplica dos grupos de estudio: Grupo experimental, y grupo control, el nivel de la investigación es de presencia y ausencia de la variable independiente. El esquema que se plantea es el siguiente:

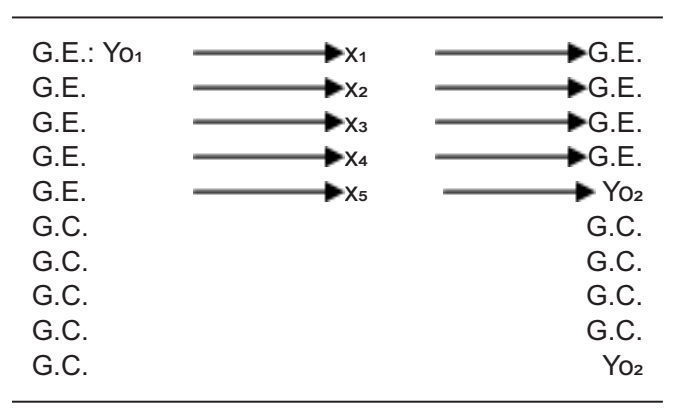

Figura1. Modelo de experimentación

Donde:

-G.E.: Grupo experimental.

G.C.: Grupo control.

$\mathrm{YO}_{1}, \mathrm{YO}_{2}$, Evaluación de progreso al grupo experimental.

$\cdot Y^{\prime} \mathrm{O}_{1} \mathrm{Y}^{\prime} \mathrm{O}_{2}$ Evaluación de progreso al grupo control.

$\cdot Y$ : Actitudes frente a la matemática.

$\cdot \mathrm{X}_{1}, \mathrm{X}_{2}, \mathrm{X}_{3}, \mathrm{X}_{4} \mathrm{y} \mathrm{X}_{5}$ : Sesiones de Historia de la Matemática

La unidad de análisis constó de estudiantes del primer ciclo de la Escuela Profesional de Medicina de la Universidad Continental - Lima en el semestre académico 2019-II.

En cuanto a la población, estuvo conformada por 34 estudiantes del primer ciclo de la Escuela Profesional de Medicina de la Universidad Continental - Lima en el semestre académico 2019-II.

La operacionalización de las variables se presenta a continuación: 
Tabla 1. Actitudes frente a la matemática

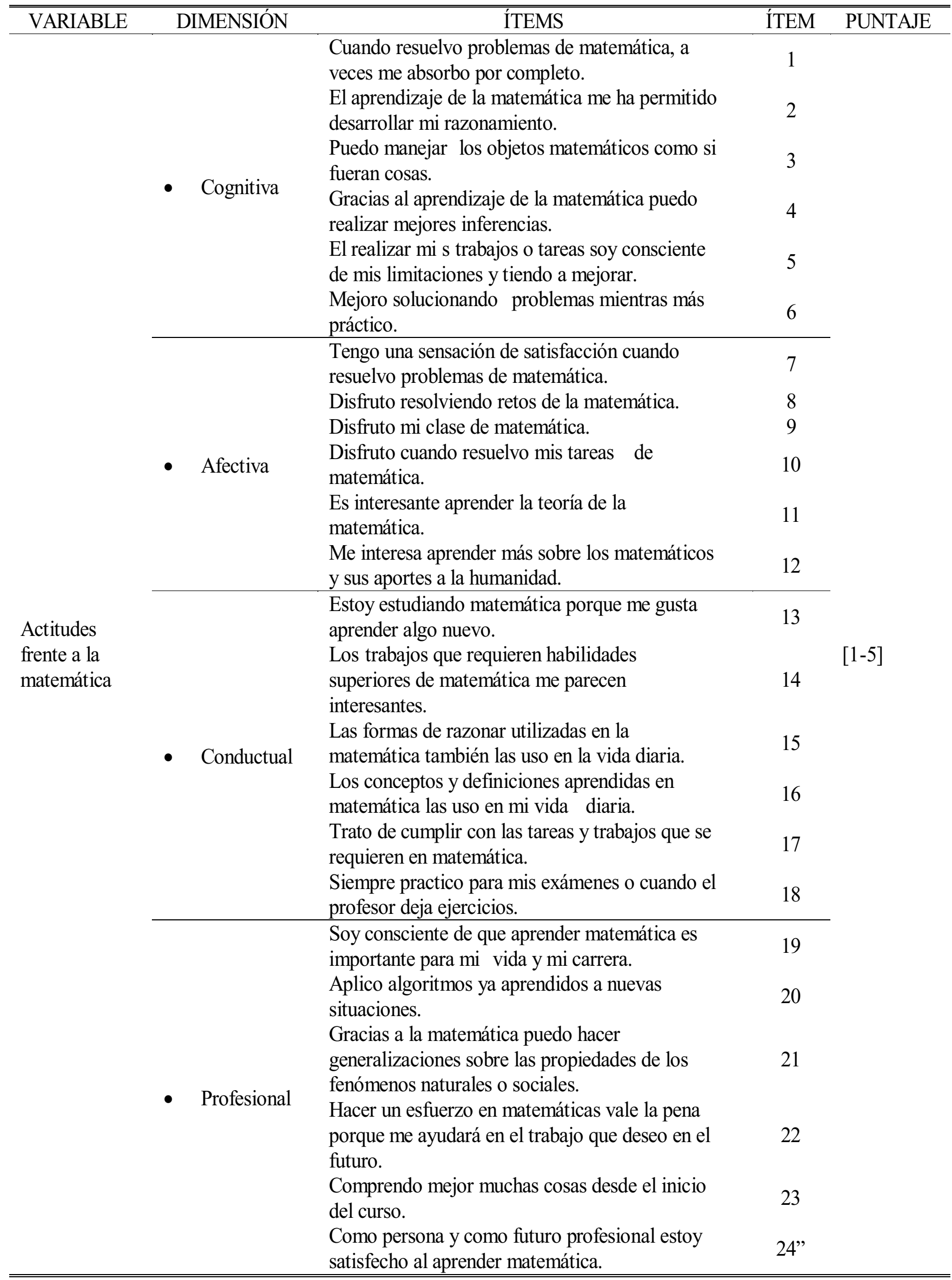


Tabla 2. Historia de la Matemática

\begin{tabular}{llll}
\hline \hline \multicolumn{1}{c}{ VARIABLE } & ACCIÓN & \multicolumn{1}{c}{ ACCIÓN } & \multicolumn{1}{c}{ TEMÁTICA } \\
\hline & & $\begin{array}{l}\text { Historia de la teoría } \\
\text { conjuntos }\end{array}$ \\
& & Historia de los números \\
Historia de la & Presencia & 5 sesiones & Historia de las funciones \\
Matemática & & Los problemas griegos. \\
& & & Las paradojas matemáticas \\
\cline { 2 - 4 } & Ausencia & Ninguna & No aplica \\
\hline
\end{tabular}

\section{RESULTADOS}

La investigación se llevó a cabo en la Universidad Continental Lima. Se evaluó a los estudiantes del primer ciclo de la Escuela Profesional de Medicina en el semestre académico 2019-II.
A continuación, se presentan los principales estadísticos para para poder comprobar los resultados agregando el coeficiente de variación:

Tabla 3. Estadísticos del grupo control - pretest - actitudes frente a la matemática (Y)

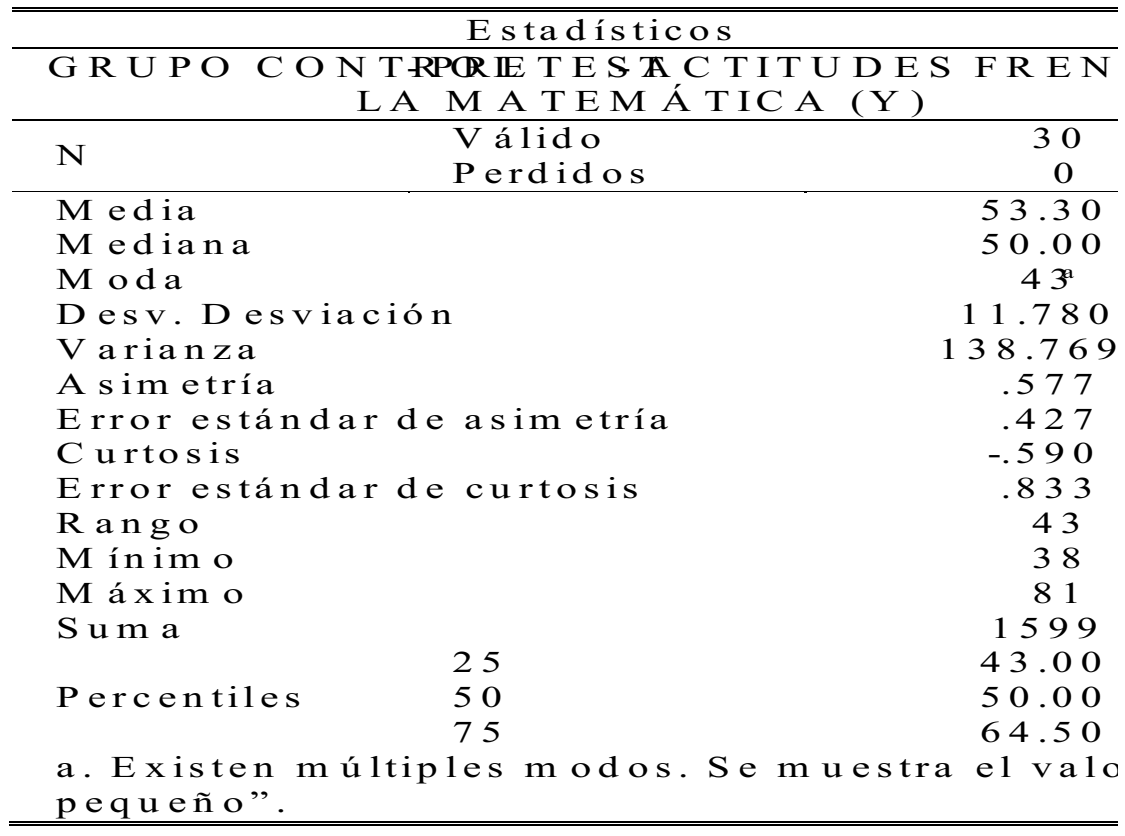

Tabla 4. Frecuencias del grupo control - pretest - actitudes frente a la matemática (Y).

\begin{tabular}{|c|c|c|c|c|c|}
\hline \multicolumn{6}{|c|}{ “GRUPO CONTROL- PRETEST- ACTITUDES FRENTE A LA MATEMÁTICA (Y) } \\
\hline & & Frecuencia & Porcentaje & $\begin{array}{l}\text { Porcentaje } \\
\text { válido }\end{array}$ & $\begin{array}{l}\text { Porcentaje } \\
\text { acumulado }\end{array}$ \\
\hline \multirow{4}{*}{ Válido } & {$[24 ; 48>$ Bajo } & 15 & 50.0 & 50.0 & 50.0 \\
\hline & {$[48 ; 72>$ Medio } & 13 & 43.3 & 43.3 & 93.3 \\
\hline & {$[72 ; 96>$ Alto } & 2 & 6.7 & 6.7 & 100.0 \\
\hline & Total & 30 & 100.0 & 100.0" & \\
\hline
\end{tabular}




\section{GRUPO CONTROL - PRETEST - ACTITUDES FRENTE A LA MATEMÁTICA ( ()}

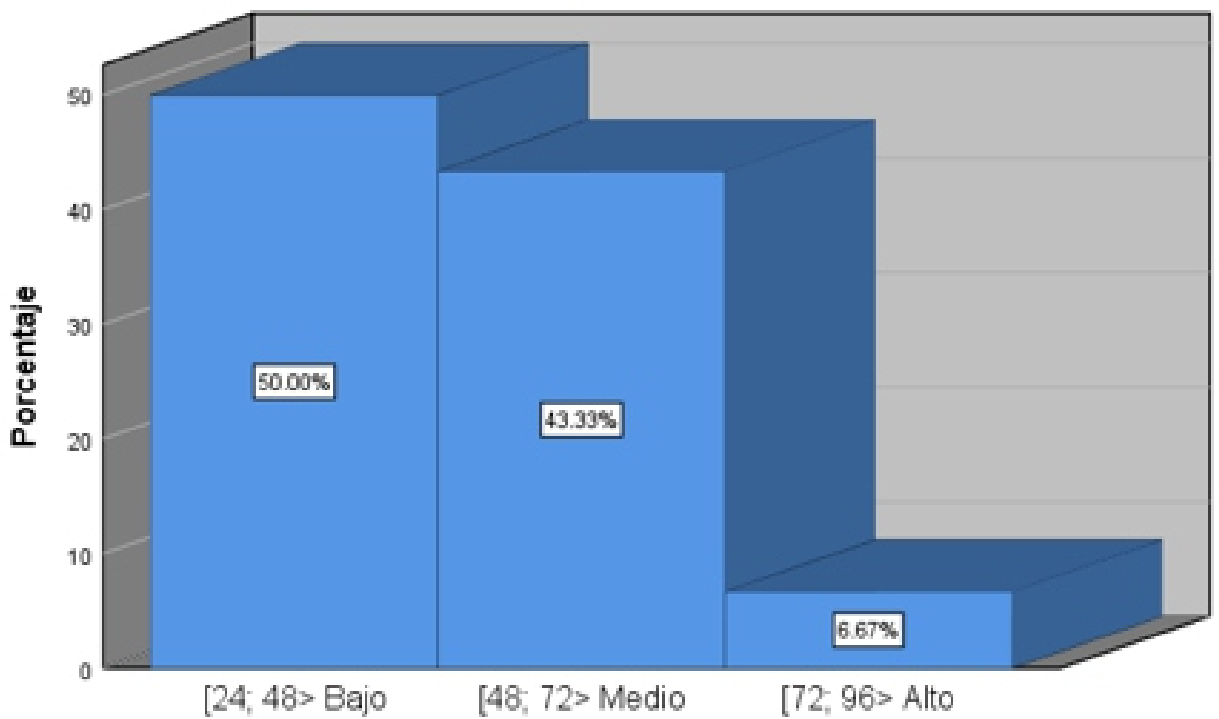

VARIABLE: ACTITUDES FRENTE A LA MATEMÄTICA $M$

Figura 2. Frecuencias del grupo control - Pretest - actitudes frente a la matemática $(\mathrm{Y})$

Según se puede apreciar; en el gráfico y en las tablas anteriores para el Prestes del Grupo Control de la variable Actitudes Frente a la Matemática $(Y)$ se puede notar que gran parte de los estudiantes, tienen un $50 \%$ considerado como bajo en el intervalo [6; 12>. Seguido de un $43,3 \%$ en el intervalo [12; 18> que considera que en un nivel medio. Luego se puede observar que un $6,7 \%$ con un intervalo [18; $24>$ considera que esta dimensión es de un nivel alto. A continuación, se evidencia que el $0 \%$, con un intervalo $[24 ; 30]$ que, tiene un nivel de desarrollo muy alto. Finalmente, el porcentaje de $0 \%$ en un intervalo [0; 6> considera esta situación que ocurre en un nivel muy bajo. Además, los estadísticos de tendencia central toman valores $53,3,50$ y 43 a que son la media, mediana y moda respectivamente.

Tabla 5. Estadísticos del grupo control - postest - actitudes frente a la matemática (Y)

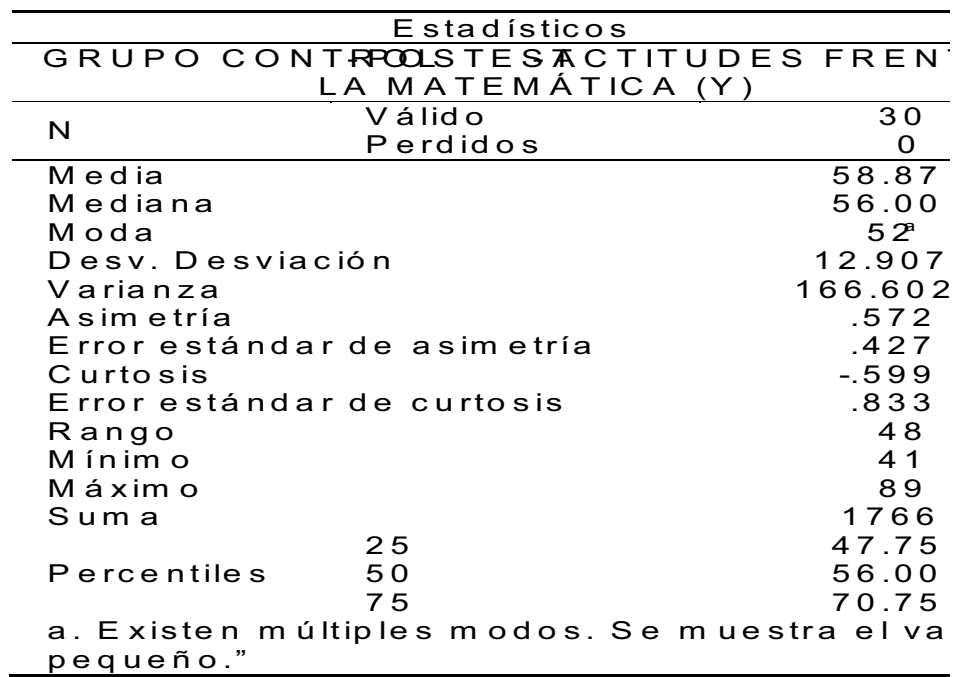

\begin{tabular}{|c|c|c|c|c|c|}
\hline \multicolumn{6}{|c|}{ “GRUPO CONTROL- POSTEST - ACTITUDES FRENTE A LA MATEMÁTICA (Y) } \\
\hline & & Frecuencia & Porcentaje & Porcentaje válido & $\begin{array}{l}\text { Porcentaje } \\
\text { acumulado }\end{array}$ \\
\hline \multirow{3}{*}{ Válido } & {$[24 ; 48>$ Bajo } & 8 & 26.7 & 26.7 & 26.7 \\
\hline & {$[72 ; 96>$ Alto } & 7 & 23.3 & 23.3 & 100.0 \\
\hline & Total & 30 & 100.0 & 100.0" & \\
\hline
\end{tabular}




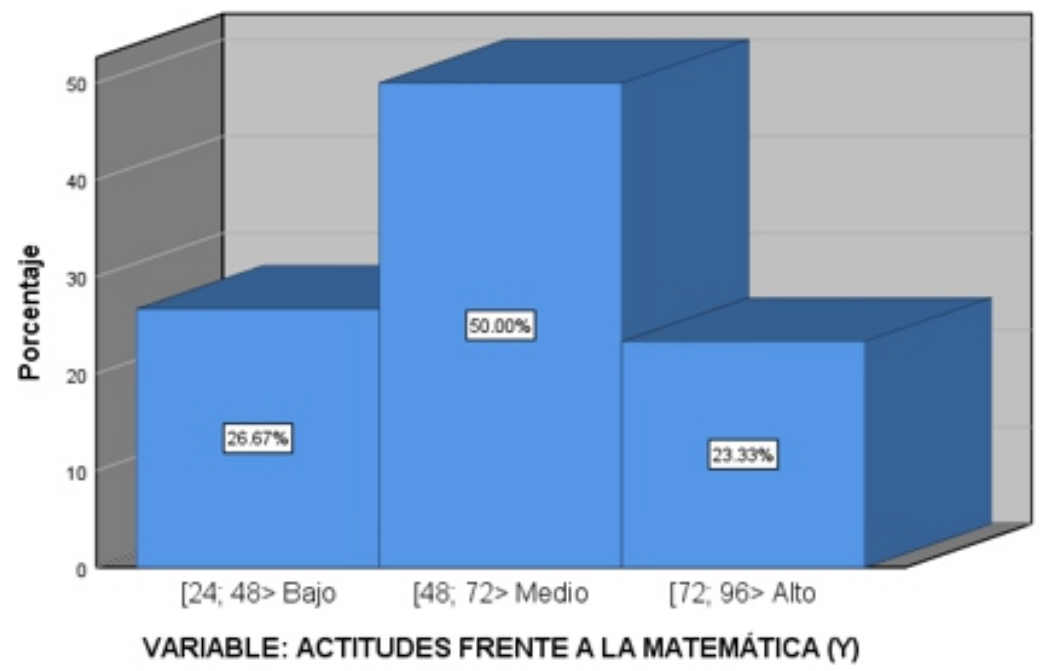

Figura 3. Frecuencias del grupo control - postest - actitudes frente a la matemática $(Y)$

Según se puede apreciar; en el gráfico y en las tablas anteriores para el Postest del grupo control de la variable Actitudes Frente a La Matemática $(\mathrm{Y})$ se puede notar que gran parte de los estudiantes, tienen un $50 \%$ considerado como medio en el intervalo [12; $18>$. Seguido de un $26.7 \%$ en el intervalo [6; 12> que considera que en un nivel bajo. Luego se puede observar que un $23.3 \%$ con un intervalo [18; 24> considera que esta dimensión es de un nivel alto. A continuación, se evidencia que el $0 \%$, con un intervalo [0; 6> que, tiene un nivel de desarrollo muy bajo. Finalmente, el porcentaje de $0 \%$ en un intervalo $[24 ; 30]$ considera esta situación que ocurre en un nivel muy alto. Además, los estadísticos de tendencia central toman valores $58.87,56$ y 52 a que son la media, mediana y moda respectivamente.

\section{DISCUSION}

La comprobación de las hipótesis muestra una diferencia entre el grupo control y el grupo experimental, se ha visto cómo se ha comprobado las hipótesis, ahora se hará un análisis más relacionado al desarrollo observando las diferentes comparaciones usando el coeficiente de variación (CV) y gráficos de comparación.

Tabla 7. Comparación de estadísticos

“Estadísticos

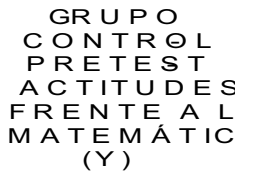

GR P P O EXPERIM EN-TA PRETEST ACTITUDES MATEMÁTICA
GRUPO CONTR O POSTEST A C TITU DE FRENTE A L M A TEMÁTIC ( $Y$ )
GRUPO

EXPERIMEN-T A POSTEST ACTITUDES MATEMÁTI\&AA

\begin{tabular}{|c|c|c|c|c|c|}
\hline \multirow{2}{*}{$\mathrm{N}$} & V álid o & 30 & 30 & 30 & 30 \\
\hline & P erdidos & 0 & 0 & 0 & 0 \\
\hline \multicolumn{2}{|l|}{ M e dia } & 53.30 & 52.40 & 58.87 & 79.57 \\
\hline \multicolumn{2}{|l|}{ Mod a } & $43^{3}$ & 53 & $52^{a}$ & 55 \\
\hline \multicolumn{2}{|c|}{ Desv. Desviación } & $11.78 c$ & $16.11 i$ & $12.90 i$ & $24.29 \leqslant$ \\
\hline \multicolumn{2}{|l|}{ A sim e tría } & 0.577 & -0.093 & 0.572 & -0.090 \\
\hline \multicolumn{2}{|c|}{$\begin{array}{l}\text { Error estándar de } \\
\text { asimetría }\end{array}$} & 0.427 & 0.427 & 0.427 & 0.427 \\
\hline \multicolumn{2}{|c|}{ Curtosis } & -0.590 & -0.594 & -0.599 & -0.566 \\
\hline \multicolumn{2}{|c|}{ Error estándar de cul } & 0.833 & 0.833 & 0.833 & 0.833 \\
\hline \multirow{3}{*}{ Percentiles } & 25 & 43.00 & 38.50 & 47.75 & 59.25 \\
\hline & 50 & 50.00 & 53.00 & 56.00 & 80.00 \\
\hline & 75 & 64.50 & 64.50 & 70.75 & 97.75 \\
\hline
\end{tabular}




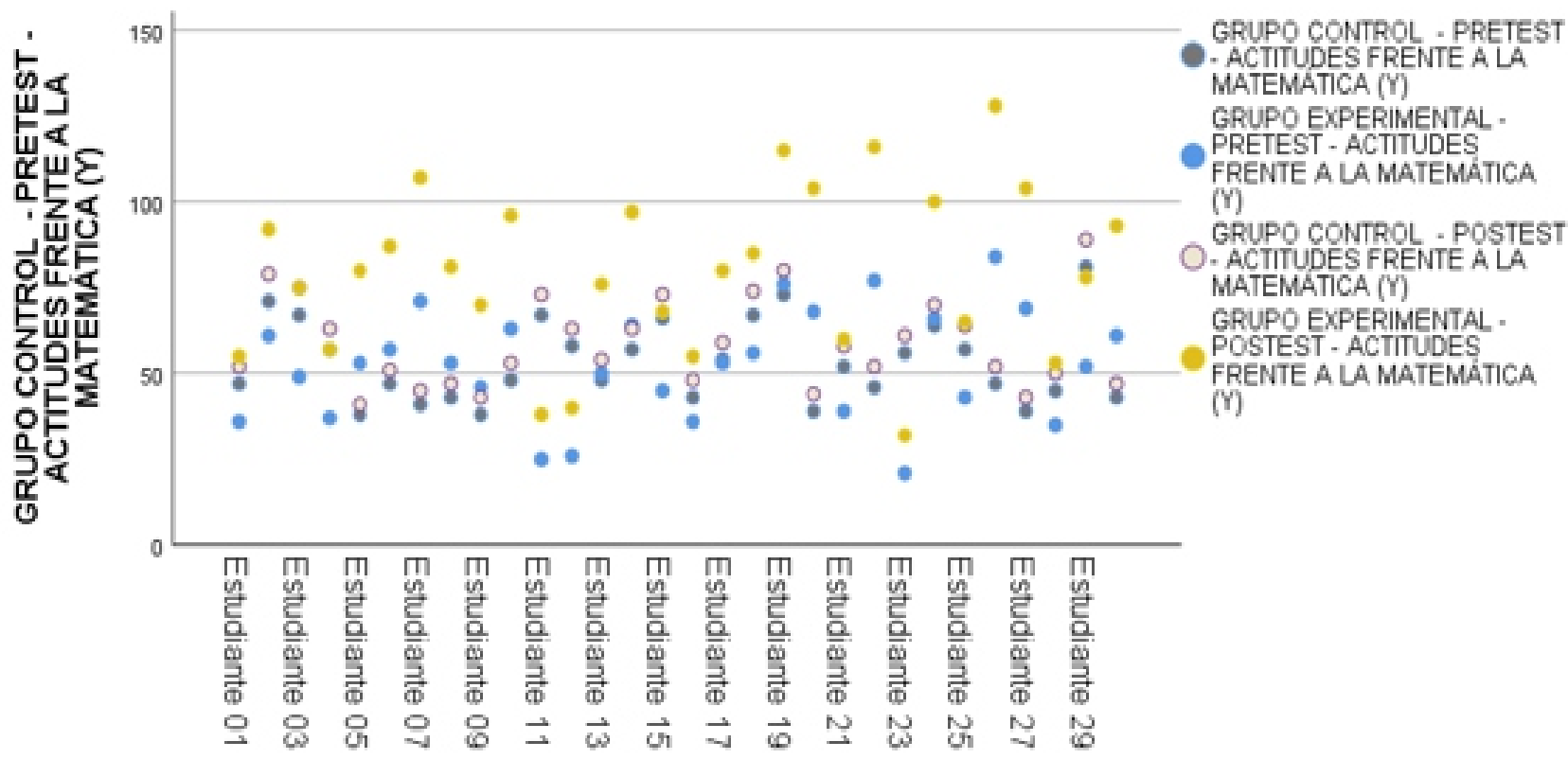

Alumnos

Figura 4. Comparación entre grupos

La tabla y el gráfico mostrados anteriormente muestran datos muy interesantes, al comprar la homogeneidad de los grupos de control y experimental respecto de la variable Actitudes Frente a la Matemática, el grupo control en el postest muestra una mayor desarrollo, es decir, que es este grupo que es más homogéneo debido a que su coeficiente de variación es menor ( $C V=21.930)$ este resultado se puede mal interpretar, el hecho de que sea homogéneo no quiere decir que tenga el mayor valor, esto no es verdad el hecho un grupo que sea homogéneo señala que los resultados se parecen entre sí, sin embargo, no necesariamente estos tiene los mayores valores. Por otro lado, las medias muestran que el grupo experimental luego del experimento tiene una media 79.57 , por lo tanto, es donde hay un desarrollo cuantitativamente superior es el grupo experimental. Como se puede mostrar en el gráfico de los puntos más altos toma la variable.

También se puede apreciar que el grupo con nivel de homogenización más bajo es el grupo experimental en pretest con un coeficiente de variación de $30.760 \%$, seguido por el mismo grupo experimental, pero en postest, mostrando que se mantiene la homogenización por medio de la experimentación, se puede evidenciar el efecto que de la historia de la matemática en los estudiantes, es decir que no solo llega permitir el desarrollo de las actitudes frente a la matemática, si no que mantiene las diferencias.

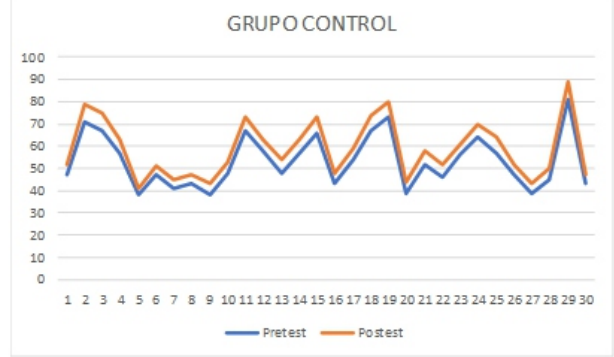

Figura 5.
Se puede apreciar que el grupo control mantiene las características en las dos evaluaciones, el motivo de que el desarrollo del grupo control sea significativo se debe a la exposición de las clases de matemática y la sugestión por una calificación han influenciado de manera positiva sobre la Actitudes frente a la Matemática. En el grupo experimental se puede apreciar que existe una celebración en los valores de manera uniforme y simétrica como se puede apreciar en el siguiente gráfico.

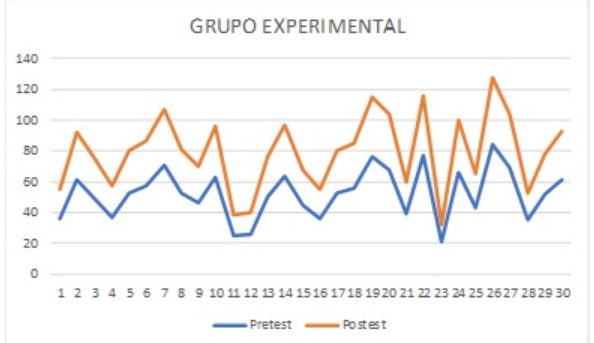

Figura 6. Comparación grupo experimental

\section{CONCLUSIONES}

La Historia de la Matemática influye directamente sobre las Actitudes frente a la Matemática de una manera significativa, positiva en los estudiantes de la Escuela Profesional de Medicina de la Universidad Continental - Lima en el semestre académico 2019-II con un coeficiente de significancia bilateral de 0.000 .

La Historia de la Matemática influye directamente sobre la dimensión Cognitiva de la variable Actitudes frente a la Matemática de una manera significativa, positiva en los estudiantes de la Escuela Profesional de Medicina de la Universidad Continental - Lima en el semestre académico 2019-II con un coeficiente de significancia bilateral de 0.000 .

La Historia de la Matemática influye directamente sobre la dimensión Afectiva de la variable Actitudes frente a la Matemática de una manera significativa, positiva en los 
estudiantes de la Escuela Profesional de Medicina de la Universidad Continental - Lima en el semestre académico 2019-II con un coeficiente de significancia bilateral de 0.000 .

La Historia de la Matemática influye directamente sobre la dimensión Conductual de la variable Actitudes frente a la Matemática de una manera significativa, positiva en los estudiantes de la Escuela Profesional de Medicina de la Universidad Continental - Lima en el semestre académico 2019-II con un coeficiente de significancia bilateral de 0.000 .

La Historia de la Matemática influye directamente sobre la dimensión Profesional de la variable Actitudes frente a la Matemática de una manera significativa, positiva en los estudiantes de la Escuela Profesional de Medicina de la Universidad Continental - Lima en el semestre académico 2019-II con un coeficiente de significancia bilateral de 0.000 .

El grupo control también tiene un desarrollo significativo debido a la influencia de las clases de la matemática, porque lo que se puede afirmar que por medio de las clases de la matemática efectuadas de manera tradicional también se puede desarrollar las Actitudes frente a la Matemática.

\section{AGRADECIMIENTOS}

Agradezco a Dios por la vida, a mis padres por apoyo y amor incondicional permanente, a mis hermanos y a Silvia, mi novia de quien siempre siento su apoyo y ánimo. A mis colegas de la maestría con quienes compartí gratos momentos y en especial al Dr. Agripino García Armas un excelente maestro de la universidad.

\section{REFERENCIA BIBLIOGRAFICA}

Gómez, M. I. (2009). Actitudes matemáticas: propuestas para la transición del bachillerato a la universidad. Educación Matemática, 21(3), 5-32.

González, P. M. (2004). La historia de las matemáticas como recurso didáctico e instrumento para enriquecer culturalmente su enseñanza. Suma, 45, 17-28.

González, P. M. (2004). La historia de las matemáticas como recurso didáctico e instrumento para enriquecer culturalmente su enseñanza. Suma, 45, 17-28.

Mamani, O. (2012). Actitudes hacia la matemática y el rendimiento académico en estudiantes del $5^{\circ}$ año de secundaria: Red N 7 Callao. Lima.Mato, D., Soneira, C., \&

Muñoz, J. M. (2018). Estudio de las actitudes hacia las Matemáticas en estudiantes universitarios. Números, 97, 7-20.

Temple, E. (2016). La historia de las matemáticas como recurso didáctico e instrumento para enriquecer culturalmente su enseñanza (Vol. 45). México: Mc Graw Hill.

Temple, E., \& Puig, A. (2004). La historia de las matemáticas como recurso didáctico e instrumento para enriquecer culturalmente su enseñanza. Revista Suma, 17-28.

Vidal, R. (2003). La historia de la matemática y su incorporación en el aula una síntesis de algunas propuestas. Santiago. 